\title{
Nonlinear Magneto-Electric Response of Giant Magnetostrictive-Piezoelectric Composite Sensors Subjected to Harmonic and Colored Noise Excitation
}

\author{
Jia Xu, ${ }^{1,2}$ Yingxiao Kong, ${ }^{1}$ Hongli Wang, ${ }^{1}$ and Zhiwen $\mathrm{Zhu}^{{ }^{*}}$ \\ ${ }^{1}$ Department of Mechanics, Tianjin University, Tianjin 300072, China \\ ${ }^{2}$ Tianjin Key Laboratory of Nonlinear Dynamics and Chaos Control, Tianjin 300072, China
}

(Received February 27, 2015; accepted January 14, 2016)

Keywords: giant magnetostrictive materials, piezoelectric ceramics, hysteretic nonlinearity, magnetoelectric response, colored noise

Nonlinear magneto-electric response characteristics of a giant magnetostrictive-piezoelectric composite sensor subjected to harmonic and colored noise magnetic excitation are discussed in this paper. A nonlinear dynamic model for such a sensor has been developed. The expression of the system's dynamic response was obtained, and the bifurcation conditions were determined. Finally, the effects of the system parameters on the dynamic characteristics were analyzed. The results of numerical simulation and experiments show that the stochastic noise intensity has an important influence on the system's dynamic response, and the stochastic resonance phenomenon occurs as the stochastic noise intensity varies. The results of this study are helpful for achieving the optimal design and improvement of giant magnetostrictive-piezoelectric composite sensors.

\section{Introduction}

The past few years have seen an increasing focus on research on giant magnetostrictivepiezoelectric composite sensors. This sensor has considerable potential for measuring magnetic field intensity (MFI). It is highly sensitive, simple in structure, low in cost, and can be used to measure low-intensity magnetic fields.

Many researchers have studied magnetostrictive-piezoelectric laminate composites and magnetostrictive-piezoelectric sensors. The magnetoelectric properties of piezoelectricmagnetostrictive laminate composites were first studied by Ryu et al. ${ }^{(1)}$ Giant magnetoelectric effects in ferroic composites of rare-earth-iron alloys were calculated by Nan and Li. ${ }^{(2)}$ Longitudinal and transverse magnetoelectric voltage coefficients of magnetostrictive-piezoelectric laminate composites were analyzed by Dong et al. ${ }^{(3)}$ The magnetoelectric behavior of Terfenol-D composite and $\mathrm{Pb}-\mathrm{Zr}$-Ti (PZT) ceramic laminates were studied by Nersessian et al. ${ }^{(4)}$ In the sensor field, magnetoelectric effects in magnetostrictive-piezoelectric composites combined with flux concentration effects in sensitive magnetic sensors were improved by Zhang et al., ${ }^{(5)}$ and the nonlinear dynamic characteristics of giant magnetostrictive sensors were studied by Zhu et al. ${ }^{(6)}$ Although many achievements in magnetostrictive-piezoelectric composite structure have been made in previous

*Corresponding author: e-mail: zhuzhiwen@tju.edu.cn 
years, there are few theoretical results on the dynamic characteristics of the magnetostrictivepiezoelectric composite sensors because of their complex nonlinear characteristics. ${ }^{(7-14)}$

The purpose of a giant magnetostrictive-piezoelectric composite sensor is to measure the magnetic field. However, there is always electromagnetic interference (EMI) from the environment, which can be regarded as a stochastic magnetic excitation in the sensor. In this report, hysteretic models of the inverse piezoelectric effect in piezoelectric ceramics and the magnetostrictive effect in giant magnetostrictive materials are both proposed, and a nonlinear dynamic model of a giant magnetostrictive-piezoelectric composite sensor subjected to harmonic and colored noise magnetic excitation is presented; the nonlinear magnetoelectric response characteristics of the system are analyzed, and the effect of the stochastic noise intensity on the system's dynamical response are discussed.

\section{Nonlinear Dynamic Model of Giant Magnetostrictive-Piezoelectric Sensor}

The structure of a giant magnetostrictive-piezoelectric composite sensor is shown in Fig. 1. The composite sensor is made of giant magnetostrictive material (Terfenol-D film), a polyimide substrate, and PZT piezoelectric ceramics. The MFI-strain curve of Terfenol-D is shown in Fig. 2.

The MFI-strain curve of Terfenol-D shows hysteresis. In this paper, the Van der Pol hysteretic model is applied to describe the Terfenol-D's MFI-strain curves as

$$
\varepsilon=e_{1} H+\left(e_{2} H-e_{3} H^{2}\right) \dot{H},
$$

where $\varepsilon$ is the strain, $H$ is the MFI, and $e_{i}(i=1-3)$ are the coefficients determined by the hysteretic loop.

The displacement-voltage curves of PZT are shown in Fig. 3. Similar to Terfenol-D, the hysteretic nonlinear piezoelectric equation of PZT can be shown as

$$
D=\varepsilon_{33}^{s} E_{3}+k_{1} E_{3}^{3}+\left(k_{2} E_{3}-k_{3} E_{3}^{2}\right) \dot{E}_{3},
$$

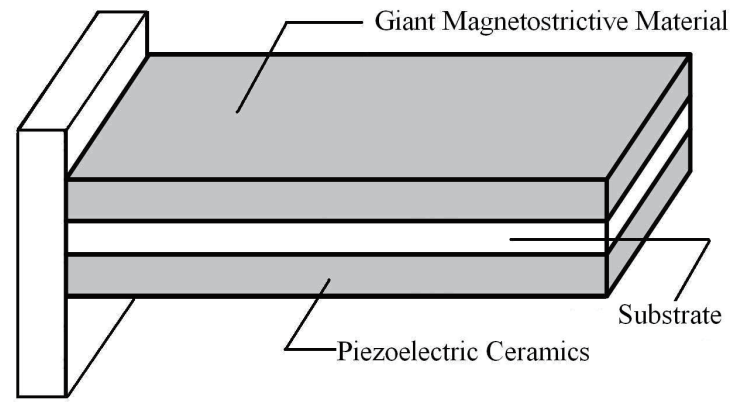

Fig. 1. Structure of magnetostrictive-piezoelectric composite sensor.

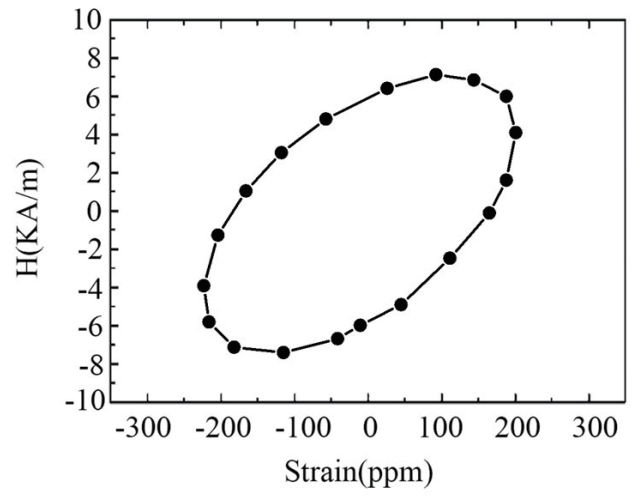

Fig. 2. MFI-strain curve of Terfenol-D. 


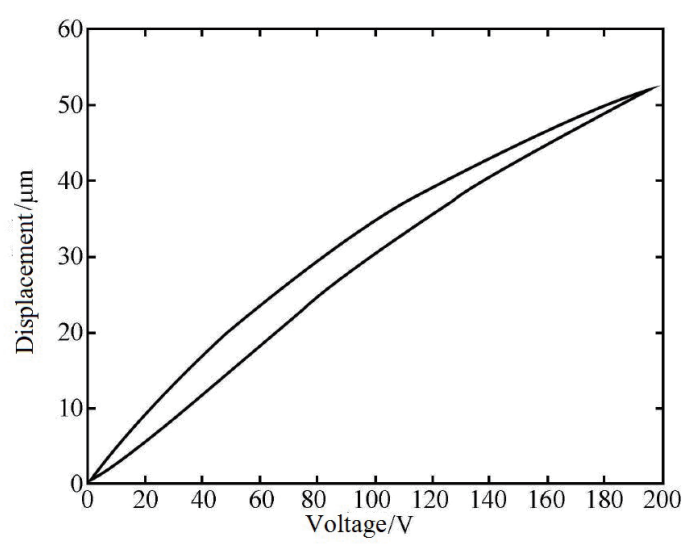

Fig. 3. Displacement-voltage curves of PZT.

where $D$ is the electric displacement, $E_{3}$ is the electric field intensity, $\varepsilon_{33}^{s}$ is the dielectric coefficient, and $k_{i}(i=1,2)$ are the coefficients.

Thus, the dynamic model of the system subjected to harmonic and colored noise magnetic excitation can be shown as follows: ${ }^{(15)}$

$$
m \frac{\partial^{2} u}{\partial t^{2}}+\left(c+\int_{0}^{l} E_{2} A \varepsilon d x\right) \frac{\partial u}{\partial t}-a_{1} \frac{\partial^{2} u}{\partial x^{2}}-a_{2} \frac{\partial^{4} u}{\partial x^{4}}-a_{3} \int_{0}^{L} E_{3} D d x=\frac{1}{2}\left(E_{2} A_{2} \frac{\partial^{2} u}{\partial x^{2}}+\rho_{2} A_{2} \frac{\partial^{2} u}{\partial t^{2}}\right)
$$

where $m=\rho_{1} A_{1}+\frac{1}{3} \rho_{2} A_{2}, a_{1}=\frac{E_{2} A_{2}}{3}+\frac{\varepsilon_{33}^{s} E_{1} A_{1}}{2}, a_{2}=\frac{k_{1} A_{2}}{8 L}$, and $a_{3}=\frac{A_{1}}{4 L}$.

The vibration mode for cantilever composite sensors is $u(x, t)=\psi(x) y(t)$, where $\psi(x)=\sin \lambda_{i} x-$ $\sinh \lambda_{i} x+\bar{\alpha}_{i}\left(\cosh \lambda_{i} x-\cos \lambda_{i} x\right), y=y(t)$ is the amplitude of system response, and $\cos \lambda_{i} L \cosh \lambda_{i} L+1=0$, $\bar{\alpha}_{i}=\frac{\sinh \lambda_{i} L+\sin \lambda_{i} L}{\cosh \lambda_{i} L+\cos \lambda_{i} L}$. The dynamic equation of system response can be solved using Eq. (3) by Galerkin's method as

$$
\ddot{x}=2 \eta \dot{x}+c_{1} x+c_{2} x^{3}+\left(c_{3} x-c_{4} x^{2}\right) \dot{x}=b \sin [\Omega t+\chi+\sigma B(t)],
$$

where $2 \eta=\frac{c}{m}, \quad c_{1}=\frac{4 \pi^{2} a_{1} L^{2}-\pi^{4} a_{2}}{16 m L^{4}}, c_{2}=\frac{\pi^{4} a_{3}}{32 m L^{3}}, c_{3}=\frac{a_{3} k_{2}}{4 m L^{2}}, c_{4}=\frac{a_{3} k_{3}}{8 m L^{3}}, b=\frac{\bar{H}}{4 m L^{2}}, \Omega$ is the center frequency, $\chi$ is the uniformly distributed random phase between $[0,2 \pi), \sigma$ is the intensity of $B(t)$, and $B(t)$ is the standard Wiener process.

\section{Dynamic Characteristics of the System}

If we let $p=x=A \cos (\omega \Theta)$ and $q=\dot{x}=-A \omega \sin (\omega \Theta)$, then we can obtain the averaged Ito equation for Eq. (4) using the stochastic average method as follows: 


$$
\left\{\begin{array}{l}
d A=m_{1}\left(A, \Delta^{\prime}\right) d t \\
d \Delta^{\prime}=m_{2}\left(A, \Delta^{\prime}\right) d t+\sigma d B(t),
\end{array}\right.
$$

where $A$ and $\Delta^{\prime}$ are two-dimensional diffusion processes.

$$
\begin{gathered}
\Delta^{\prime}=\Omega t+\sigma B(t)+\chi-\Theta, \\
m_{1}\left(A, \Delta^{\prime}\right)=\frac{\pi}{4} c_{4} A^{4}-\frac{b A}{2 \omega} \cos \Delta^{\prime}, \\
m_{2}\left(A, \Delta^{\prime}\right)=2 \pi \Omega-\frac{1}{\eta}\left(\frac{3}{4} \pi c_{2} A^{3}-\frac{b}{2} \sin \Delta^{\prime}\right) .
\end{gathered}
$$

The averaged Fokker-Planck-Kolmogolov (FPK) equation for the probability density $f=f\left(A, \Delta^{\prime}, t\right)$ is as follows:

$$
\frac{\partial f}{\partial t}=-\frac{\partial}{\partial A}\left(m_{1} f\right)-\frac{\partial}{\partial \Delta^{\prime}}\left(m_{2} f\right)+\frac{\sigma^{2}}{2} \frac{\partial^{2} f}{\partial \Delta^{\prime 2}}
$$

The numerical results of the system response and phase diagram are presented in Fig. 4, where $\eta$ $=0.9, c_{2}=100, c_{4}=0.7$, and $\Omega=30 \mathrm{~Hz}$. As the figures show, the stochastic noise intensity $\sigma$ has an important influence on the system's dynamic response. After the stochastic noise intensity reaches a certain value, the system's response decreases as the stochastic noise intensity increases, and the stochastic resonance phenomenon occurs in the process.

The experimental results for a giant magnetostrictive-piezoelectric composite sensor subjected to harmonic and colored noise magnetic excitation are shown in Figs. 5 and 6. We also note that the system's response decreases as the stochastic noise intensity further increases beyond a certain level.

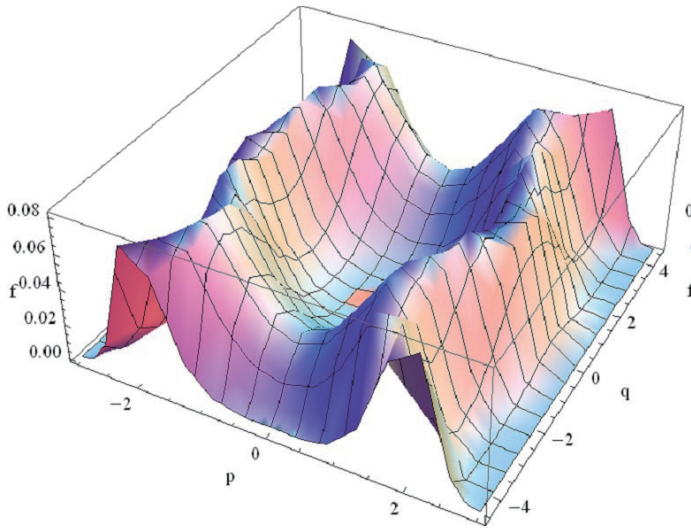

(a)

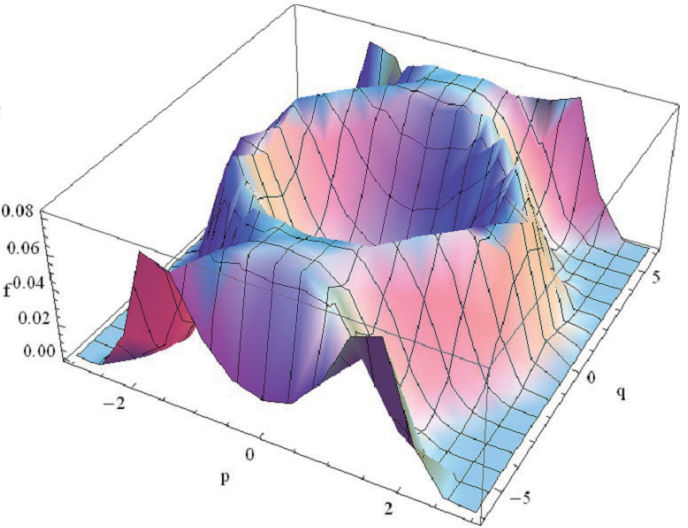

(b)

Fig. 4. (Color online) Probability density of the system's response when (a) $\sigma=0.5$ and (b) $\sigma=0.8$. 


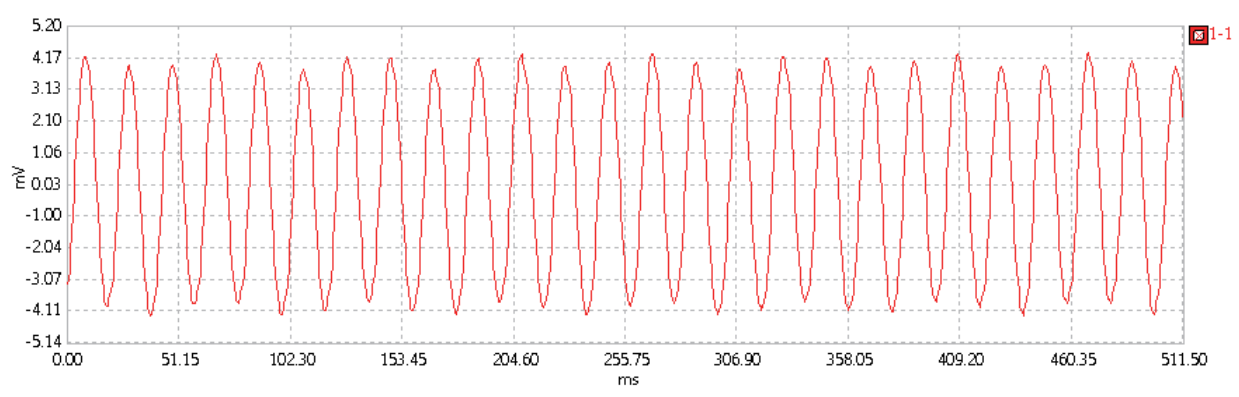

Fig. 5. (Color online) Output voltage of the composite sensor when $\sigma=0.5$.

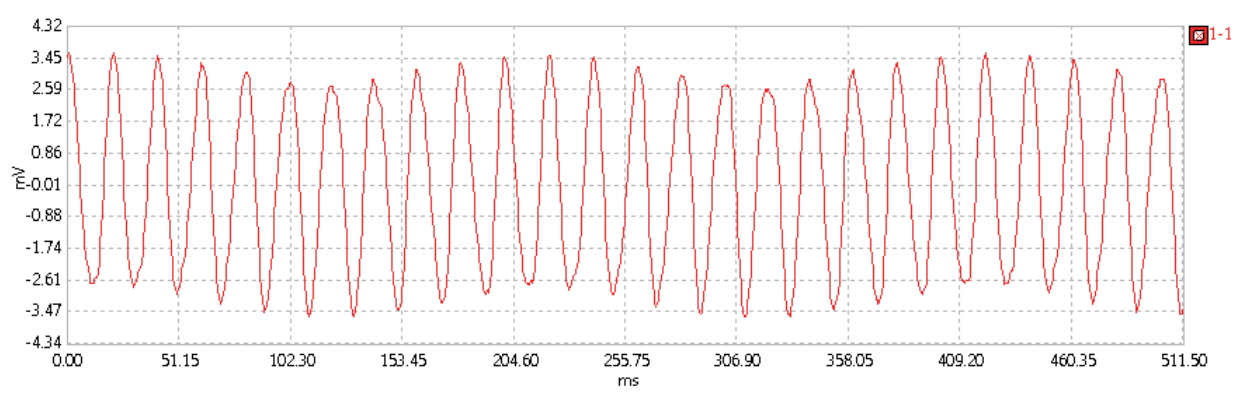

Fig. 6. (Color online) Output voltage of the composite sensor when $\sigma=0.8$.

\section{Conclusions}

The nonlinear magnetoelectric response characteristics of a giant magnetostrictive-piezoelectric composite sensor under harmonic and colored noise magnetic excitation are reported in this paper. Nonlinear differential items were introduced to explain the hysteretic behavior of the sensor, and the nonlinear dynamic model of the sensor was developed. The new models have a simple form and are easy to analyze. The dynamic response of the system may be obtained. The results of numerical simulation and experiments show that the stochastic noise intensity has an important effect on the system's dynamic response, and the system's response decreases as the stochastic noise intensity further increases beyond the level when the stochastic resonance phenomenon occurs. The results of this study may be helpful for the optimal design and improvement of giant magnetostrictive-piezoelectric composite sensors.

\section{Acknowledgements}

The authors gratefully acknowledge the support of the Natural Science Foundation of China (NSFC) through Grant Nos. 11272229 and 11302144, and the Tianjin Research Program of Application Foundation and Advanced Technology through Grant Nos. 16JCYBJC18800 and 14JCQNJC05300. 


\section{References}

1 J. Ryu, A. V. Carazo, and K. Uchino: J. Appl. Phys. 40 (2001) 4948.

2 C. W. Nan and M. Li: Phys. Rev. B 63 (2001) 144415.

3 S. X. Dong, J. F. Li, and D. Viehland: IEEE Trans. Ultrason. Freroelectr. Freq. Control 50 (2003) 1253.

4 N. Nersessian, S. W. Or, and G. P. Carman: IEEE Trans. Magn. 40 (2004) 2646.

5 H. Zhang, C. J. Lu, and C. B. Xu: AIP Adv. 5 (2015) 047114.

6 Z. W. Zhu, W. D. Zhang, and J. Xu: Sens. Mater. 26 (2014) 319.

7 D. A. Fillippov: Phys. Solid State 47 (2005) 1080.

8 P. Li, Y. M. Wen, and L. X. Bian: Appl. Phys. Lett. 90 (2007) 022503.

9 V. M. Petrov, G. Srinivasan, and M. I. Bichurin: Phys. Rev. B 75 (2007) 224407.

10 C. S. Lee, J. Joo, and S. Han: Appl. Phys. Lett. 85 (2004) 1841.

11 Z. Y. Jia, W. Liu, and Y. S. Zhang: Sens. Actuators, A 128 (2006) 158.

12 Q. X. Yang, H. Y. Chen, and S. Z. Liu: IEEE Trans. Magn. 42 (2006) 939.

13 S. Masuda, Y. Matsumura, and Y. Nishi: J. Jpn. Inst. Met. 70 (2006) 166.

14 N. Tiercelin, V. Preobrazhensky, and P. Pernod: J. Magn. Magn. Mater. 210 (2000) 302.

15 Z. W. Zhu, Q. X. Zhang, and J. Xu: Chin. Phys. B. 23 (2014) 088201. 